\title{
Dans le noir. La pièce qu'on ne voit pas (Bonbon acidulé de Ricardo Sued )
}

Antonia García Castro et Estelle Durand

\section{(2) OpenEdition}

\section{Journals}

Édition électronique

URL : http://journals.openedition.org/conflits/3132

DOI : $10.4000 /$ conflits.3132

ISSN : $1777-5345$

Éditeur :

CCLS - Centre d'études sur les conflits lilberté et sécurité, L'Harmattan

Édition imprimée

Date de publication : 15 novembre 2007

Pagination : 177-188

ISBN : 978-2-296-04582-8

ISSN : 1157-996X

Référence électronique

Antonia García Castro et Estelle Durand «Dans le noir. La pièce qu'on ne voit pas (Bonbon acidulé de Ricardo Sued ) », Cultures \& Conflits [En ligne], 67 | automne 2007, mis en ligne le 04 janvier 2010, consulté le 30 mars 2021. URL : http://journals.openedition.org/conflits/3132 ; DOI : https://doi.org/ $10.4000 /$ conflits.3132 


\title{
Dans le noir. \\ La pièce qu'on ne voit pas (Bonbon acidulé de Ricardo Sued ${ }_{1}$ )
}

\author{
Antonia GARCIA CASTRO, Estelle DURAND ${ }^{2}$
}

\author{
«EUGÉNIE: Eteins toutes les lumières, que tout reste \\ dans l'ombre. \\ MARIO : Pourquoi ? \\ EUGÉNIE : Après je t'explique. \\ MARIO : ... c'est fait. \\ EugÉNIE : Viens, je veux te dire quelque chose. (Dans \\ l'obscurité de l'obscurité, Mario rejoint Eugénie). Et si \\ tout cela était un rêve ? [...] \\ MARIO : Je ne sais pas, tout cela quoi ? \\ EUGÉNIE : La vie, tout. ${ }^{3}$ »(p. 14)
}

$\mathrm{O}$ ne voit pas. La pupille se dilate. Si la salle est grande ou petite, on l'ignore. Cela non plus, on ne le voit pas. Je suis assise entre deux vieilles dames. A ma droite, une amie. A gauche, une inconnue. Je l'ai vue dans le hall

1. Sued R., Bonbon acidulé, Paris, Actes Sud, 1999. Traduit de l'espagnol (Argentine) par Dominique Poulange. Titre original : Caramelo de limón. La pièce fut d'abord montée en Argentine (1991) puis à Paris (1996).

2 . NDR : Ce dossier donne à entendre plusieurs voix, celle de l'auteur de la pièce (R. Sued), celles de ses personnages (Eugénie, María et les autres), celle d'une spectatrice (A. García Castro), celle d'un comédien et metteur en scène (G. Reyna). Un mot semble nécessaire sur les conditions de travail car celles-ci renvoient, en partie, à la pièce elle-même et à sa spécificité la plus évidente (elle se déroule dans l'obscurité et, selon le mot d'Estelle Durand, traite « de la force de la réminiscence qui se joue des lieux, des espaces et qui trouve dans cette obscurité ambiante un pouvoir de suggestion des plus puissants»). Tandis que la «spectatrice » préparait son récit en mobilisant ses souvenirs et sans disposer du texte de la pièce, la « lectrice », E. Durand qui, elle, n'a pas vu la pièce, lisait et relisait, puis préparait une sélection d'extraits en fonction des thèmes qu'il nous importait d'évoquer (dans la prolongation des questions traitées dans le précédant dossier, consacré à Jill Magid ; voir Cultures E Conflits n66, été 2007). Lorsque l'assemblage de ces deux premiers éléments a été fait (souvenirs et extraits), de petites «erreurs " sont devenues évidentes (là où A. Garcia C. se souvenait d'un homme, E. Durand, texte à l'ap- 
d'entrée du théâtre de la Colline, avant d'entrer. On nous a faites entrer ensemble à la «queue leu leu », en nous tenant par l'épaule (par file de cinq). C'est ainsi qu'on nous a conduites dans la salle plongée dans le noir le plus complet dès notre arrivée. Aucune idée donc de sa dimension, de la disposition des sièges, de l'emplacement d'une scène éventuelle. La vielle dame, l'inconnue, entrée en même temps que nous, assise sur ma gauche, me parle. Elle dit : «mademoiselle... est-ce que ça vous gênerait que je vous tienne la main ? Je ne suis pas rassurée ». Cela ne me gêne pas. Je prends la main qu'on me donne. Je la garderai pendant une bonne partie du spectacle. Mais qu'est-ce que c'est que ce spectacle?

Ce que l'on sait, c'est que la pièce de théâtre s'appelle Bonbon acidulé, qu'elle a été écrite et mise en scène par Ricardo Sued et que, de ce spectacle, toute lumière a été bannie. Forcément, on ne voit pas. On attend.

«LA PETITE MARIA : Je rallume.

MARIO : Attends ! Je veux d'abord te montrer quelque chose. Ferme les yeux...

LA Petite MARIa : Ça y est.

MARIO : Bon, dis-moi ce que tu vois.

LA PETITE MARIA : Je te vois toi qui me parles...

MARIO : Maintenant, ouvre-les : ... Alors ? Tu vois quoi ?

LA PETITE MARIA : L'obscurité. » (p. 35)

Et en attendant, il est permis de poser des questions. Pourquoi dit-on qu'on va ou qu'on est allé voir une pièce de théâtre ? Pourquoi donc - s'agissant d'aller au théâtre, et non pas de lire une pièce - la question qui fait sens, c'est : «avez-vous $v u$ du Shakespeare ? " et non pas, par exemple, «avez-vous entendu du Shakespeare ? " ? D’où vient ce primat du visuel dès qu'il est question de représentation ? Puisque rien n'empêche les apprentis sociologues de se rendre au théâtre pendant qu'ils rédigent des articles sur des sujets « autres », et toujours en attendant, la relation s'impose d'elle-même, un peu lourde, un peu rustre, mais incontournable tout de même : que vaudrait le supplice dans une

pui, disait clairement qu'il s'agissait d'une femme...). Nous avons cru bon - peut-être à tort d'harmoniser, d'unifier. Et d'un échange à l'autre, d'une précision à l'autre, il est apparu que le travail sur ce dossier, dans ces différentes étapes se faisait, lui aussi, par tâtonnements. Comme si aucun des éléments donnés n'avait été tout à fait donné. Comme si même dans le dialogue, puis dans l'écriture, dans le montage du dossier autour de cette pièce, nous avions été dans le noir, prenant appui les uns sur les autres : sur la vision des autres, sur l'impression des autres, et aussi, sur leur parole. Remerciements particuliers à Miriam Perier et à Guido Reyna pour leur collaboration.

3 . Toutes les citations de ce dossier sont, sauf mention contraire, extraites de la pièce de Sued R., Bonbon acidulé, op. cit. 
société d'aveugles ? Pourquoi faut-il que l'on voit ? Quel type de relation s'établit par le regard ? Et que devient cette relation lorsque le regard ne suit plus, ne se pose plus? Ne regarde plus? Cesse-t-on de regarder parce qu'on ne voit plus ? (Ou est-ce l'inverse ?) Cesse-t-on de voir parce qu'on ne regarde plus ?

Une voix. Le spectacle commence. Chose inouie, la voix ne vient pas de "là-bas », de ce lieu censé se trouver en face des «spectateurs ", qu'on appelle habituellement une scène. Elle vient de derrière. L'actrice qui parle, on l'a dans le dos. On se tourne. Les yeux ouverts ont leurs habitudes. Ils veulent voir la femme. Ils ne la verront pas. La tête tout entière revient à sa place. On écoute. On ne peut pas faire autrement. La femme s'avance, continue de parler, s'approche, nous dépasse. S'en va. Et on ne sait plus, du tout, où l'on est.

Tout au long de la pièce, il en sera de même. L'histoire, elle, se déroule, autour de cette femme revenue dans la maison de son enfance, de ses souvenirs, de ses évocations. D’autres personnages se présentent, parlent, se parlent. Il est question d'amour. C'est une histoire d'amour qui se raconte. Puis, autre chose. Une absence. Une absence soudaine et inexpliquée. Une disparition. De celles que l'on dit «forcées ». Cette histoire, on l'entend. Mais il y a plus. Elle nous touche. Non pas (seulement), elle nous «émeut ». Elle nous frôle.

Par exemple, lorsqu'ils sont à la piscine. Ils disent - les personnages - qu'ils sont à la piscine. Ils parlent fort car il y a un bruit de fond, des rires, des bavardages inaudibles, l'écho, le brouhaha particulier des piscines. Ils plongent. (On les entend nettement plonger), nagent, font une longueur. Les voilà, le bruit de l'eau brassée. Ils arrivent, passent devant nous, devant moi et là... je reçois des éclaboussures en pleine figure! Je lâche la main de la vieille. Je m'essuie le visage. Je le touche. Je vérifie. C'est de l'eau. C'est vraiment de l'eau.

On se redresse sur son siège. On sent bien le mouvement de la rangée. L'émotion. Mais qu'est-ce que c'est que cette pièce ?

Jusqu'à ce qu'on s'habitue. Il y a un moment, sans doute différent pour chacun (peut-être n'est-ce pas d'ailleurs le cas de tout le monde), où on s'habitue.

Ils sont là et ailleurs. Ils sont parmi nous. On n'est pas en face d'une scène, on n'assiste pas à une pièce. On est dans la pièce. On occupe l'entre-deux. L'espace qui sépare les acteurs les uns des autres; l'espace qui les sépare des choses, meubles présumés et plantes, par exemple; oui, les plantes et leur bruissement dans cette petite boutique, où se passent des choses importantes à cause de la pépiniériste. La rencontre de deux femmes - dont la pépiniériste qui aiment le même homme, et ne le savent pas.

Puis, cet autre moment de la pièce, le rêve. Un drôle de rêve. Le rêve d'un homme. Des personnages à la voix bizarre, parlent et s'écrient. On les sent 
gigoter, circuler. Il est question de bonbons acidulés. Ils sont venus en distribuer dans le rêve. Derrière, devant, de côté, de partout, et, soudain, le bonbon est dans ma main. Que faire ? Que peut-on faire d'un bonbon ? (J'enlève l'emballage. Je mange le bonbon).

On n’est plus étonné. On ne s’étonne plus.

Les yeux ouverts ou fermés, des images prennent place. Et si elles peuvent prendre place c'est qu'on a déjà vu. Paroles, bruits et mouvements permettent à chacun de composer une scène possible, un décor, et aussi des physionomies, forcément singuliers selon les personnes présentes, les « spectateurs ». Chacun mobilise son expérience, convoque intérieurs et extérieurs, visages et silhouettes vus ou entraperçus. De telle manière qu'en sortant du théâtre, chacun aura assisté à la même pièce et à une pièce différente.

\begin{abstract}
"MARIA : Mon père aimait beaucoup ce livre... "... il y a un principe de magie, une qualité vivante, en toute chose. En tous lieux, toujours, quelque chose d'intense, quelque chose de beau est en train de se produire..." Mon père me racontait que chaque fois qu'il lisait ce livre, son adolescence et le Gitan lui revenaient à la mémoire. »(p.15).
\end{abstract}

Des années plus tard, certaines réflexions que ce spectacle a fait naître reviendront autrement, influencées ou non (comment savoir ?) par cette pièce qu'on n'a pas vue. Noir sur blanc, il sera question d'un supplice invisible, de cette capacité du pouvoir à soustraire, à cacher la scène de la violence la plus crue, lorsqu'elle s'abat sur les corps et sur autre chose que les corps, comme c'est le cas des disparitions forcées ${ }^{4}$, que cette pièce évoque avec subtilité, et de tout ce que les disparitions forcées engagent eu égard aux logiques discrètes ${ }^{5}$ dont usent parfois les agents de l'Etat. Il est troublant de remarquer que l'absence radicale de lumière aux fins de divertissement - sans qu'en aucun cas le mot revête ici un sens péjoratif - puisse miser, que cela soit voulu ou non, sur deux éléments essentiels sur lesquels repose aussi la scène discrète de la violence organisée aux fins de meurtre et de contrôle : l'homme-spectateur, l'homme-sujet, l'homme-citoyen, l'homme-tout-court se souvient et imagine, il est doté de mémoire et d'imagination. Il sait ce qu'il sait et imagine ce qu'il pourrait savoir. Il le conçoit.

4. Voir les numéros «Disparitions", Cultures $\mathcal{E}$ Conflits, n¹3-14, 1994 et « Survivre », Cultures $\mathcal{E}$ Conflits, $\mathrm{n}^{\circ} 24-25,1996$.

5. Voir l'article de Bigo D., «Disparitions, coercition et violence symbolique ", Cultures E Conflits, n¹3-14, op. cit., pp. 3-16. 
«Dehors, des coups violents aux portes et aux fenêtres : on veut entrer de force.

Des VoIX AutORItaIRES : Ouvrez ! Ouvrez !

AleXANDRA : (les militaires viennent l'arrêter. Elle a vingt et un ans).

Mario... ! Comme je voudrais que tu sois là. J'ai si peur... !

Il y a tant d'obscurité dans tout cela.

Pourvu que le monde change un jour...

Pourvu que le monde change un jour...

Pourvu que le monde change...

Pourvu...

Pourvu... » (pp. 13-14)

Lorsque, enfin, la lumière s'allume et que viennent les applaudissements, on reste tout à fait penaud. C'était donc ça! Ils sont là, maintenant, où les acteurs sont censés être, devant les spectateurs, en justaucorps, semblables les uns aux autres, impossible de dire qui joue quel rôle. Les personnages se sont évanouis. La scène intérieure, elle, s'est éteinte. Parce qu'on a vu.

\section{Entretien avec Ricardo Sued - extraits 6}

\section{D’où est venue l'inspiration de Bonbon acidulé ?}

J'avais entendu dire qu'il y avait des gens qui travaillaient dans le noir, au niveau de la méditation. L'idée m'a semblé intéressante et je me suis dit pourquoi ne pas le faire au théâtre, en supprimant l'élément vital, la lumière ? [...]

«Faisons une histoire d'amour dans le noir » ai-je dit [aux acteurs], et dès la première improvisation nous avons été fascinés, par les possibilités de ce travail.

$Y$ a-t-il eu une différence entre les réactions du public (en Argentine et en France)?

Là-bas (à Paris) ça a été incroyable. Ici la pièce était conçue pour peu de gens, trente, quarante, ou cinquante personnes, parce qu'il y avait une relation assez directe avec le public. Au Théâtre national de la Colline on m'a dit : « quarante personnes... laisse tomber ». Et on l'a montée pour deux cents spectateurs. Les réactions ont été exactement les mêmes. [...]

6. Publié le 8 novembre 2006 sur le site Internet : «Lavoz.com.ar ». La version intégrale est disponible, en espagnol, sur :

http://www.lavoz.com.ar/herramientas/imprimir_nota.asp?nota_id=16070. Cet entretien a été traduit de l'espagnol par A. García C. 
Nous sommes tellement habitués à dominer, à tout contrôler par la vision. Sans cela, tu te sens démuni. Dans le noir, il y a deux options : tu te fermes complètement, comme une personne qui a peur, ou tu relâches la pression et alors tu peux t'ouvrir d'une manière incroyable. Tout arrive plus vite au cœur.

La pièce parle d'une absence, il y a un lien avec la dictature 7 , encore récente en 1991.

L'histoire est liée à cette période mais ce n'est pas un témoignage politique, bien qu'elle aborde la question de la dictature et d'un « disparu ». Le rôle de la mort est important, associé au noir, mais la pièce ne devient pas dépressive parce qu'elle est comme un chant d'amour. Paradoxalement, dans un monde avec tellement de lumière, à travers l'image, le divertissement, la question de la mort, elle, est cachée. Les gens ne veulent rien savoir de la mort. L'obscurité ouvre la question de la mort, c'est pour cela que nous avons voulu l'évoquer; c'est un monde si réel, il apparaît d'un instant à l'autre. C'est une structure solide, semblable à notre monde, et un petit rien suffit à la faire s'écrouler, comme dans un rêve.

\section{Variations autour du «Bonbon ». Entretien avec Guido Reyna, acteur et metteur en scène 8}

\section{CEC : Pourrais-tu me parler de tes impressions à propos de Bonbon acidulé ?}

G.R. : L'expérience de «voir » la pièce de Ricardo Sued, Bonbon acidulé, à ses débuts, à Córdoba aux alentours de 1992, a eu pour moi une grande importance à des niveaux différents. Je voudrais préciser qu'à cette époque j'entamais mon travail d'acteur, en travaillant aussi bien sur des scènes officielles (La Comedia Cordobesa) que sur des scènes liées à des pratiques plus alternatives, disons, plus «underground»; par ailleurs, à cette époque, je me formais également en tant que spectateur.

La première impression est, évidemment, l'effet quelque peu déstabilisateur du fait qu'on est privé de la vue, à cela s'ajoute, immédiatement, le parfum du bonbon acidulé (au citron, en l'occurrence ${ }^{9}$ ) comme un appel à l'univers de l'enfance. Cette sensation se prolonge, ensuite, avec l'expérience du toucher, puisque certains personnages jouent de la proximité physique et de la sensua-

7. Référence à la dictature argentine de la période 1976-1983.

8. Guido Reyna, acteur et metteur en scène d'origine argentine, réside actuellement à Paris, membre fondateur de la compagnie «Isn't It » (www.isntit.fr), avec laquelle il a présenté, entre autres Les Bâtisseurs d'Empire ou le schmürz de Boris Vian, Erzebeth de C. Prin, Kâma-sûtra : observations générales à partir des textes de Vâtsyâyana (théâtre à domicile). En 2004, il traduit et met en scène, en Argentine, L'Homme orange de L. Martin (El fruto probibido, Cie. Las Miserables). Il prépare actuellement une thèse de doctorat en psychanalyse sur « Le comédien et sa relation avec l'inconscient » (université de Paris-VIII). Entretien réalisé et traduit de l'espagnol par A. García C. depuis Buenos Aires (juin 2007).

9. Pour mémoire, le titre original de la pièce est Caramelo de limón : bonbon au citron. 
lité du contact, une sorte de lutin distribue des morceaux de chocolat et éveille de la sorte le sens du goût. Progressivement, qu'on le veuille ou non, se produit un certain abandon, ou plutôt une relativisation des barrières rationnelles de la perception et on se retrouve à la merci d'un tourbillon des sens. Ce qui n'est pas chose facile à assumer et que peu de spectacles sont capables de produire.

Un deuxième moment, que j'associe à une sorte de « déformation professionnelle », est celui des saluts. C'est là qu'on découvre les moyens si rudimentaires mis en place pour nous conduire, par exemple, dans un bar à l'heure de pointe, dans la piscine d'une maison de campagne et, même, dans les recoins d'un garde-manger où apparaît le lutin de la sieste. Pour un jeune acteur, ce moment des saluts est presque aussi surprenant que le reste. C'est un instant magique et fascinant notamment parce qu'il permet de supposer, et d'espérer, qu'au théâtre tous les univers peuvent être créés à partir de rien. Et ça c'est quelque chose qui libère complètement la fantaisie et la créativité.

Mais il existe un troisième moment où se fait place une réflexion sur notre propre pratique, sur le sens et la fonction du théâtre. Y a-t-il un message à transmettre ? Comment ? Quelle place pour l'acteur et sa propre construction imaginaire ? Etc. Je pense que la pièce de Sued s'inscrit dans une période singulière du mouvement théâtral argentin de la post-dictature. A mon sens, il s'agit d'une sorte de synthèse entre les propositions de certains dramaturges et metteurs en scène qui ont essayé d'esquiver la censure par le biais d'une construction métaphorique et celles d'autres professionnels qui, après l'avènement d'un régime démocratique, ont adopté une modalité plus documentaire, souvent didactique, brechtienne, pour rapporter les faits historiques et politiques. Avec son spectacle, Sued montre qu'il est possible de construire une fiction, de fait traversée par le contexte historique et social (par exemple, le traumatisme de la dictature) et que pour la raconter, le mieux c'est de partir de ce qui est moins évident, moins « visible », mais probablement plus réel, l'intuition.

CEC: Tu parles d'un effet destabilisateur, provoqué par l'absence de lumière qui, d'une certaine manière, serait compensé par l'éveil ou la mobilisation des autres sens. Seulement, au théâtre, celui qui assiste à la pièce est appelé, très précisément, un "spectateur». Le spectateur qui ne voit pas reste-t-il un spectateur?

G.R. : Effectivement, je crois qu'au-delà de l'effet à la fois destabilisateur et mobilisateur que la pièce de Sued suscite au niveau sensoriel et intellectuel, on ne peut s'empêcher de penser à la manière dont se situe le spectateur au sein de ce nouveau système esthétique. Si on se rapporte à ce qui a été l'une des modifications fondamentales du théâtre au XXe siècle, notamment à partir d'une vision contestataire et anti-système des années 1960 et 1970 - l'abolition du quatrième mur - on se rend compte qu'au théâtre, comme dans l'art en général, l'enjeu est toujours de pouvoir formuler des alternatives à la vision prédomi- 
nante du monde. L'évolution de l'idée consistant à faire disparaître la séparation entre la scène et la salle, grande invention d'Antonin Artaud (qui nous semble aujourd'hui aussi banale qu'à l'époque elle a semblé radicale), a surtout prouvé que le problème est moins de détruire complètement les cadres de référence que de se les approprier et de les transformer. En d'autres termes, le fait que les acteurs se mêlent physiquement au public n'empêche pas la différence élémentaire qui fait du théâtre une construction, une lecture de la réalité. Ce qui est différent ici c'est la possibilité donnée au spectateur d'organiser son propre spectacle. Dans ce sens, je dirais que pour les spectateurs de Bonbon acidulé la question n'est pas tant de "ne pas voir » mais de voir, justement, «ce qu'ils ne voient pas » ou de voir autrement, chacun selon son propre univers sensoriel. En d'autres termes, ce qui est en jeu, c'est aussi une façon de voir.

\section{CEC : Et qu'est-ce que cela implique pour un acteur d'" être vu» et de "ne pas être vu»?}

G.R. : C'est une question intéressante si l'on pense au fait que l'art même de l'acteur se structure autour du regard de l'autre, un peu comme le dit Lacan au sujet du «stade du miroir » pendant lequel l'enfant aurait la sensation que son corps est formé de parties détachées et que c'est le regard de l'autre (dans ce cas, de la mère) qui coagule sa propre image. Il me semble que cette sensation est assez proche de celle que peut ressentir un acteur, en particulier sous la forme de ce petit (parfois énorme...) vertige qui se produit entre les répétitions et le moment de la représentation. Dans ce sens, la pièce de Sued est une expérience fondamentale non seulement pour le public mais aussi pour l'acteur qui doit articuler son action à partir d'un regard « invisible» mais non pas inexistant pour autant. C'est comme si, alors même que le spectateur réinvente les images, l'acteur devait de son côté réinventer le regard, le sien et celui de l'autre. Ceci me fait penser à la pièce de Tom Stoppard, Rosencrantz et Guildenstern sont morts. Il s'agit de deux amis de Hamlet auxquels le roi a donné l'ordre de surveiller ce dernier. Stoppard a structuré sa pièce comme s'il s'agissait du côté caché : Hamlet ne joue qu'un petit rôle alors que ses deux amis sont les personnages principaux, et l'action même se situe à des moments que le spectateur n'est pas censé voir, en « hors-champ ». Il y a un passage fantastique où l'un des acteurs dit à Guildenstern :

«Vous ne comprenez pas cette humiliation : nous voir privés de la seule hypothèse qui rende viable notre existence : être regardés par quelqu'un... Nous sommes des acteurs... nous avons mis nos identités en gage, confiant dans cette convention de notre métier que quelqu'un nous regarde, et puis, peu à peu, personne 10 » (Acte II). 
CEC : Y a-t-il une scène de Bonbon acidulé qui, en tant que spectateur, t'aurait interpellé plus que les autres?

G.R. : Peut-être la scène de la piscine.

CEC : Pourrais-tu donner quelques détails?

G.R. : Je ne pense pas être capable de donner beaucoup de détails, mais dans mon souvenir, $j$ 'associe le réalisme des brassées du personnage qui nage et fait des longueurs, tandis qu'une "voix off» décrit ses pensées, ses sentiments. Il me semble que c'est une des scènes qui concentre un maximum de stimuli: le sentiment tactile de l'eau et le son du choc du corps lorsqu'il plonge, l'image d'une énorme piscine, la voix du personnage qui est aussi une voix intérieure et (pourquoi pas ?) le souvenir de l'odeur d'eau de javel... qui est sans doute une invention de mon cru, en tant que spectateur. Mais c'est aussi l'une des scènes où l'on se sent le plus distant. Comme si les détails captés par la perception étaient absorbés par ceux de l'émotion, le vertige de ne pas pouvoir calculer la distance qui nous sépare, ou nous confond, avec le personnage et ses circonstances.

CEC : Ceci est lié à ce que tu disais sur l'espace théâtral en tant que tel; parce que ce qui est effacé ici ce n'est pas seulement la représentation en tant que telle, mais aussi la place traditionnellement attribuée au public (et aux acteurs); cette place, dans Bonbon acidulé, est une sorte de non-lieu, dans le sens où elle n'a pas de coordonnées spatiales. Le spectateur ne sait pas où il est situé par rapport aux acteurs. Il peut en avoir l'intuition à partir des données sensorielles que tu évoquais. Y a-t-il une spécificité de Bonbon acidulé de ce point de vue ? Disons, concernant cette question de la distance entre acteurs et spectateurs et la manière dont le spectateur participe de la pièce?

G.R. : La singularité de la pièce de Sued est précisément moins dans le fait d'effacer la représentation (il s'agit toujours d'une pièce de théâtre, d'une fiction...) que dans la perte de la dimension de l'espace, produite par le noir. Le fait est que le regard est le cadre de référence par excellence sur lequel se fonde le dispositif théâtral. Il me semble que le mérite particulier de cette pièce est plutôt dans l'inversion de la hiérarchie sensorielle. Concernant la distance entre acteurs et spectateurs comme paramètre, la manière dont ces derniers prennent part à la pièce, je crois qu'il y a toujours eu des expériences, plus ou moins élaborées, dans ce sens. Par exemple, la Commedia dell'arte ou le théâtre élisabéthain auquel on assistait comme à une fête foraine. Justement, cette proximité avec le public, c'est quelque chose que nous essayons d'explorer avec notre compagnie en créant, par exemple, nos spectacles à domicile. Ce sont des spectacles conçus pour des espaces modifiables et non conventionnels où les acteurs se mêlent aux spectateurs, s'adressent à eux, sans oublier leur personnage. Je crois que Bonbon acidulé se présente comme une sorte de confirmation de ce que Peter Brook appelle l'«espace vide », c'est-à-dire 
comme instrument que l'acteur peut utiliser à volonté. Dans ce cas, ce que le spectateur peut partager c'est un espace vide (vide même de lumière), comme une couverture magique sous laquelle chacun peut choisir une lecture qui lui est propre et interpréter l'action de la pièce, les motivations profondes des personnages, leur aspect, etc. C’est aussi ce côté éphémère du théâtre qui nous rappelle sans cesse qu'il y a une fissure non seulement entre la réalité et la fiction, entre le passé et le présent, la scène et les coulisses, le noir et la lumière... et que l'homme se construit dans cette fissure, ou plutôt dans cette brèche où il n'y a rien et où il y a tout... Le théâtre nous rappelle constamment cela, et l'acteur dans son travail même est obligé de faire face, à chaque représentation, à cette brèche entre ce qu'il sait ou croit savoir et ce qui se passe in situ. Il y a une phrase que Shakespeare fait dire à Hamlet (dans la célèbre scène de la pièce dans la pièce, où un groupe d'acteur a été convoqué pour mettre en scène l'assassinat de son père) qui résume brillamment la question :

" [l'acteur peut] par la pensée forcer son âme dans une fiction, dans le rêve d'une passion et tant qu'à ce travail toute sa face blêmit, que les larmes lui viennent aux yeux, qu'il s'égare, se perd, brise sa voix, toutes formes soumises à la force de ce qu'il conçoit ! Le tout pour rien, pour Hécube ! » (Acte II, sc. II).

CEC : Et soudain, la lumière... J'aimerais qu'on reprenne ce que tu disais au sujet des saluts, la pièce étant terminée, ta découverte des moyens utilisés, tes impressions à ce propos.

G.R. : Oui, le moment des saluts à la fin de la pièce m'a semblé aussi ou plus fascinant que le reste. D'abord, c'est la surprise et l'admiration lorsque tu découvres que si peu de moyens techniques ont permis de reproduire les stimuli des diverses scènes avec autant de réalisme : par exemple, ce qui dans la pièce est une énorme piscine dans laquelle l'un des personnages nage désespérément pour calmer ses souvenirs et ses angoisses, n'est qu'une toute petite baignoire, de celles qu'on utilise pour les enfants en bas âge, à la lumière des projecteurs. Dans la scène du bar-restaurant en plein service, les allées et venues des clients et des habitués étaient réalisées à partir d'une table de cuisine avec deux ou trois pots en verre et une règle en métal. Il y a là quelque chose... on y repense avec le temps et c'est comme une révélation (à laquelle, je crois, le public du XVIII e siècle et des Lumières était très sensible) : la découverte de l'artifice, le comment ça marche. Ceci a eu, et a, pour mon expérience, deux sens : l'un professionnel, pour autant qu'il s'agit d'essayer d'ouvrir les possibilités de la création et de la production ; l'autre plus idéologique, dans la mesure où cette expérience singulière de l'artifice nous confronte de manière radicale et physique (dans tous les cas sensorielle) $\mathrm{au}$ «les choses ne sont pas ce qu'elles semblent être », tout en mettant en question le « si je ne le vois pas, je n'y crois pas... ». Au théâtre il y a un texte à respecter, un espace scénique où bouger, des costumes adaptés aux personnages, tout est préfiguré et, cependant, à chaque fois, il est possible de produire des 
effets de surprise, de prendre la liberté d'improviser, de créer la magie, à condition d'avoir conscience de comment fonctionne le mécanisme de la pièce. Je crois qu'en cela le théâtre reste la meilleure métaphore du monde comme artifice et que, comme tel, nous pouvons modifier, à condition de savoir, précisément, comment ça marche.

CEC : Justement, revenons-en quelque peu à la question de la place $d u$ théâtre dans ce monde et à la relation du théâtre avec les contingences historiques. Qu'en penses-tu? Est-ce une relation qui te semble s'imposer de soi ? Une option entre autres? Je reprends une des expressions que tu as utilisées pour te demander : y a-t-il une "fonction" du théatre?

G.R. : Je pense que la fonction sociale du théâtre se définit, pour ainsi dire, dans et par la représentation elle-même. Je veux dire qu'un spectacle est presque toujours un acte politique, même si, en apparence, il n'y a pas de lien avec un contexte social particulier du point de vue du contenu ou de la forme esthétique. Ceci ne veut pas dire que tout spectacle ait une incidence directe sur les contingences historiques, mais que tout spectacle introduit, comme nous l'avons évoqué plus tôt, une construction de la réalité (ce qui arrive souvent sans qu'il y ait volonté expresse de la part de ceux qui le font : auteur, directeur, acteurs...). Il existe également un théâtre plus descriptif, ou didactique, qui cherche à mettre en évidence ces mécanismes, que ce soit dans le texte ou dans la mise en scène, extrêmement utile à la formation du citoyen, mais je ne suis pas sûr que ce théâtre-là soit le plus efficace pour provoquer des effets de changement... Parfois, et surtout au théâtre, « trop de réalité brouille la réalité ».

CEC : Est-ce pour cela que, selon ton expression, mieux vaut l'intuition? Cette intuition s'oppose-t-elle à l'explicatif ? Ou de quelle manière le théâtre participe-t-il, ou non, de la construction d'un récit intelligible sur le monde?

G.R. : Dans la continuité de ce que nous venons de dire, il me semble que l'intuition et l'explicatif ne s'opposent pas forcément mais peuvent se compléter : il y a parfois des situations auxquelles le spectateur ajoute une nuance, une lecture inespérée, le spectateur se demande si ce qu'il est en train de voir est à interpréter comme il le fait ou si cette interprétation est tout entière redevable de son propre univers personnel ; parfois l'acteur même, dans sa confrontation avec le public, découvre un nouveau sens, une perspective différente sur son personnage ou sur le texte; parfois même il peut arriver que le metteur en scène choisisse de reformuler certains aspects de sa mise en scène à partir de la rencontre et de la réaction qui se produisent pendant la représentation. C’est justement ce que je te disais sur le fait que la représentation même est un acte, que le récit qui est fait sur le monde est toujours vrai, toujours partial mais vrai. Sans aller plus loin et revenant à la pièce de Ricardo Sued, je crois que, par exemple, le «bonbon acidulé » en est une illustration. Le titre de la pièce fonctionne comme indicateur de quelque chose dont on peine à dire ce que c'est, 
mais sur lequel nous avons déjà une idée, une image, un souvenir. Cet effet semble mis en évidence (outre la marque linguistique, la parole) grâce aux stimuli sensoriels. A peine entrés dans la salle, il y a ce parfum du bonbon au citron... Ensuite, le bruit de l'emballage... La saveur quand on t'en offre un, etc. A mesure que la pièce avance, ce «bonbon » devient une référence récurrente, mobilisée à divers moments, tantôt pour accentuer tantôt pour dédramatiser telle situation. Au point qu'il se présente subtilement comme quelque chose d'à la fois très réel, très ancré dans le monde où nous vivons, ou dans lequel nous avons vécu, et très lié aussi à la fantaisie et à l'imagination de chacun. Le bonbon finit par être une sorte de métaphore de lui-même, du théâtre, du monde, de l'énigme de l'existence de l'homme. Une sorte de « mise en abyme »... C'est ce qu'avance le gnome à la fin de la pièce, le petit chantage de révéler ou non un secret en échange... d'un bonbon acidulé. Mais, quel secret ?

"MARIO : j'étais dans la chambre, je dormais [...] et tout d'un coup, j'entends des bruits à la porte. Alors... je me lève, vais ouvrir, et au lieu de rencontrer comme $d^{\prime}$ 'habitude le couloir et l'escalier... je me retrouve dans un endroit étrange, très étrange, empli de bruits bizarres, de montagnes de couleurs, des bleus... des jaunes... et de choses qui bougeaient... (Entre un gnome) - Eh !... Eh, vous ![...] quel est cet endroit ?

Le GNome : C'est le pays des gnomes. [...] Peut-être que si tu me donnes quelque chose, je pourrais te laisser entrer.

MARIO : [...] quelque chose comme quoi ?

LE GNOME : Quelque chose qui brille...

[...]

Il lui donne un bonbon. On entend le papier de bonbon que le gnome défait.

LE GNOME : ...Mm...Mm... un bonbon acidulé !

D'AUTRES GNOMES : Des bonbons acidulés !

De nombreux gnomes entrent en scène. Criant, chahutant, fous de joie, jouant et se bataillant pour les bonbons. Ils interviennent dans le public, offrant et volant des bonbons aux spectateurs. L'idéal serait que chaque spectateur reçoive un bonbon. [...]

LE GNOME : Mario...

MARIO : Oui...

LE GNOME : Je vais te dire un secret... »(p. 13).

Post-scriptum

CEC : Guido... Pour toi, les bonbons au citron ont vraiment un " parfum »?

G.R. : Bien sûr que les bonbons au citron ont un parfum... Pas pour toi ? 